\title{
Influence of some rol genes on sugar content in Nicotiana and Vaccinium
}

\author{
Tatiana Matveeva ${ }^{1, *}$,Ekaterina Berezina $^{2}$, Irina Isaeva $^{1}$, Alina Dymo $^{1}$ and Sofia \\ Sokornova ${ }^{3}$ \\ ${ }^{1}$ Saint Petersburg State University, 199034 St. Petersburg, Russia \\ ${ }^{2}$ N. I. Lobachevsky State University of Nizhny Novgorod, 603950, Nizhny Novgorod, Russia \\ ${ }^{3}$ All-Russian Institute of Plant Protection, 196608 St. Petersburg, Russia
}

\begin{abstract}
In natural conditions, insertion of Agrobacterium T-DNA into the plant genome and its subsequent transfer via sexual reproduction has been shown for several dozens of species, including species from genera Nicotiana and Vaccinium. In the framework of investigation of possible function of cT-DNA in naturally transgenic species we have shown, that increasing of expression of rolC in Nicotiana tabacum is associated with increase of amount of glucose and total sugar content. Similar trend was observed for $\mathrm{rolB} / \mathrm{C}$-like gene in Vaccinium.
\end{abstract}

\section{Introduction}

Agrobacterium-mediated transformation is the key method of genetic engineering of plants aimed to introduce novel genes into their genomes. At the same time in natural conditions, horizontal transfer of T-DNA genes into the plant genomes has been shown for several dozens of species, including species from genera Nicotiana and Vaccinium [1-4]. Such TDNA was termed as «cellular T-DNA» (cT-DNA). One of the most conserved genes in cTDNA is rolC. Its expression has been shown in Nicotiana and Linaria [5-6]. It is related somehow to sugar content [7]. Gene rolC belongs to the plast genes family [8]. The plast genes are identified by their common ancestry. They are mostly described in T-DNAs. The plast genes can modify plant growth in different ways, but the molecular basis of their function remains largely unknown. Just a few agrobacterial plast genes, including rolB, rolC, orf13 and $6 b$, have been studied in detail. The list of plast genes was significantly expanded due to the discovery of new types of cT-DNA. Some plast genes have a significant potential for applied biology, agriculture and may be used to modify the growth of crops [8]. It is believed that the natural transformation of sweet potato with plast genes contributed to its domestication [3]. An interesting plast gene resembling rolB and $\mathrm{rolC}$ features was found in Vaccinium macrocarpon [4]. It is worth noting that all previously studied by us samples of $V$. macrocarpon contain intact gene while all samples of $V$. oxycoccos contain a deletion within the plast gene. Nicotiana tabacum contains intact

\footnotetext{
*Corresponding author: radishlet@gmail.com
} 
rolC. Transgenic lines, containing an additional copy of rolC under the control of a dexamethasone-inducible promoter, were obtained based on Samsun cultivar of $N$. tabacum. It is possible to increase gene expression (as compared with the control) by adding the inductor to the cultural medium. Previously, the total sugar content and transport were studied in such plants [7].

In this study we compared the carbohydrates patterns in tobacco plants, differing in expression of the rolC gene and in Vaccinium plants, carrying intact plast gene and deleted one.

\section{Materials and methods}

Plant material was represented by 2 lines of cranberries: line GVo of Vaccinium oxycoccos L. and line GVm of Vaccinium macrocarpon Aiton; and three genotypes of tobacco: $N$. tabacum, cv. Samsun and two transgenic lines, derived from this cultivar and containing rolC of different origin under Pdex: A. rhizogenes rol C (Pdex-A4-rolC) and N. tabacum rol C (Pdex-trolC) correspondingly [7].

Plants of genera Vaccinium and Nicotiana were cultivated in aseptic culture on MS medium at $24 \mathrm{~h}$ photoperiod at $22^{\circ} \mathrm{C}$. Transgenic $N$. tabacum plants, containing rol $\mathrm{C}$ under dexamethasone-inducible promoter and control non-transgenic plants were cultivated for 7 days on MS media supplied with $10 \mu \mathrm{M}$ of dexamethasone or on MS0 media [9]. Shoots were ground in liquid nitrogen, ethanol-extracted $\left(80^{\circ} \mathrm{C}, 30 \mathrm{~min}\right)$ and analyzed by HPTLC (high-performance thin-layer chromatography). The sugar standards and extracted samples were processed on the automated HPTLC system (CAMAG, Muttenz, Switzerland). The TLC plate was developed in chloroform-acetone-methanol-acetic acid- $\mathrm{H}_{2} \mathrm{O}$ 50:20:10:10:5 $\mathrm{v} / \mathrm{v}$. The developed plate was carried out with the aniline reagent in methanol under heating. All tracks in the plate were scanned at $454 \mathrm{~nm}$ wavelength and individual $\mathrm{Rf}$ values of peaks were obtained. These data were matched with the standard. The experiment was done in three replicates. Statistical processing was performed by analysis of variance (ANOVA).

\section{Results and discussion}

Samples of Vaccinium and Nicotiana differed in total carbohydrate content and ratio. ANOVA have shown statistically significant differences in total carbohydrate content, glucose and sucrose composition among samples of Vaccinium ( $\mathrm{p} \leq 0.05)$ (fig. 1A). Analysing $N$. tabacum lines we have shown statistically significant effect $(\mathrm{p} \leq 0.05)$ of both studied factors (genotype and cultural medium composition) on the sugar content (fig 1B). Difference in sugar levels of three studied tobacco genotypes without dexamethasone treatment can be partly explained by the effect of the localization of T-DNA in the genomes. In addition, dexamethasone itself increases sugar content of tobacco plants. However, the portion of the influence of dexamethasone on the level of sugars is higher in transgenic lines. For the Pdex-trolC line, Fischer intra-class correlation coefficient is $74 \%$, for the Pdex-A4rolC line it is $49 \%$, and in non-transformed plants it is only $17 \%$. The carbohydrates patterns in tobacco plants, differing in expression of the rolC gene and in Vaccinium plants, carrying intact plast gene and deleted one, have shown some common features. Firstly, the sugar levels in the induced transgenic N. tabacum (Pdex-trolC and Pdex-A4rolC) plants were higher than in N. tabacum cv. Samsun and transgenic plants 
without induction of rolC expression $(\mathrm{p} \leq 0.05)$. In $V$. macrocarpon total sugar content is higher, than in $V$. oxycoccos. Secondly, extracts from induced transgenic plant and extracts from $V$. macrocarpon show an increased level of glucose comparing to control plants. Earlier in the literature there was an idea, that the products of the $\mathrm{rolC}$ and $\mathrm{rolB}$ genes are beta-glucosidases [11-12]. However, later it was criticized [8]. Our results can be explained by an increase of glucosidase activity in case of the expression of $\mathrm{rolC}$ and $\mathrm{rolB} / \mathrm{C}$ - like gene. However, we cannot say whether this is a direct effect of studied genes, or an indirect one.

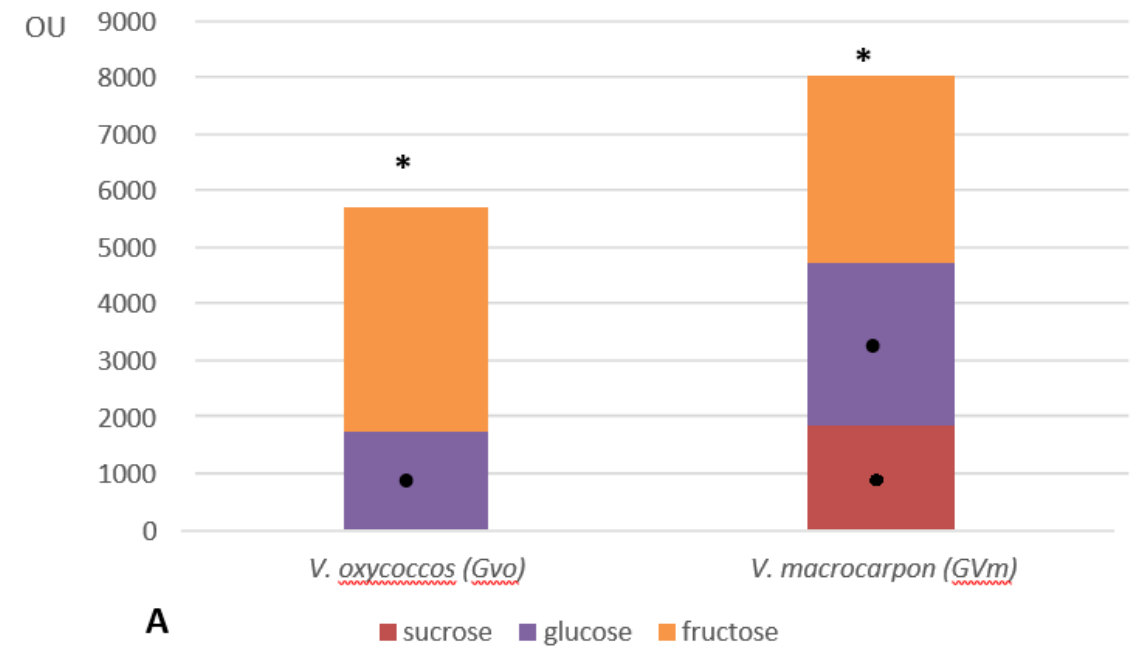

OU 14000

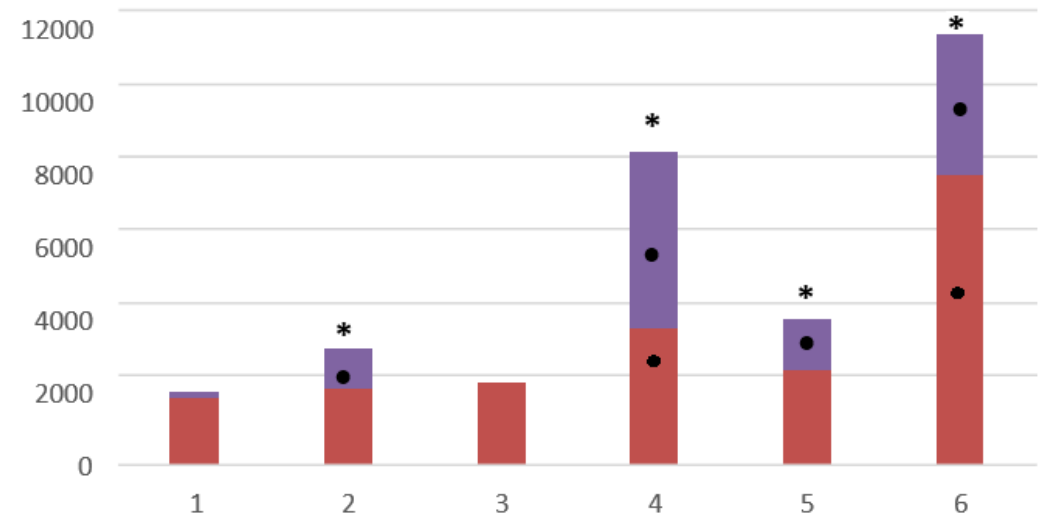

B

- sucrose glucose

Fig. 1. Sugar content of Vaccinium (A) and Nicotiana (B) plants. Significant difference $(\mathrm{p} \leq 0.05)$ for total sugar content is shown by $*$, for glucose and sucrose -

1 - Nicotiana tabacum, cv. Samsun (MSO medium), 2 - N. tabacum, cv. Samsun (MS medium+dexamethasone), 3 - N. tabacum, Pdex-trolC (MSO medium), 4 - N. tabacum Pdex-trolC (MS medium+dexamethasone), 5- N. tabacum Pdex-A4rolC (MSO medium), 6 - N. tabacum PdexA4rolC (MS medium+dexamethasone) 
This study was supported by a grant to TM from the RFBR 18-016-00118. Transgenic seeds [7] were kindly represented by Prof. L. Otten (IBMP, France).

\section{References}

1. F.F. White, D.J. Garfinkel, G.A. Huffman, M.P. Gordon, E.W. Nester, Nature, 301, 348-350 (1983)

2. T.V. Matveeva, D.I. Bogomaz, O.A .Pavlova, E.W. Nester, L.A. Lutova, Mol. Plant. Microbe Interact. 25, 1542-1551 (2012)

3. T. Kyndt, D. Quispe, H. Zhai, R. Jarret, M. Ghislain, Q. Liu, G. Gheysen, J.F. Kreuze Proc Natl Acad Sci U S A, 112(18), 5844-5849 (2015)

4. T.V. Matveeva, L. Otten, Plant Mol. Biol. 101(4-5), 415-437 (2019)

5. T. Matveeva, Curr. Top. Microbiol. Immunol. 421-441 (2018)

6. T. Matveeva, S. Sokornova Russ J Plant Physiol. 64(5), 635-64 (2017)

7. H. Mohajjel-Shoja, B. Clement, J. Perot, L. Otten, MPMI 24, 44-53 (2011)

8. L. Otten Curr, Top. Microbiol. Immunol. 418 (2018)

9. T. Murashige, F. Skoog, Physiol. Plant. 15, 165-170 (1962)

10. J.A. Bailey, R.S. Burden, G.G. Vincent, Phytochem, 14(2), 597-582 (1975)

11. J.J. Estruch, D. Chriqui, K. Grossmann, J. Schell, A. Spena, EMBO J. 10(10), 28892895 (1991)

12. J.J. Estruch, J. Schell, A. Spena, EMBO J. 10(11), 3125-3128 (1991) 